\title{
Alcohol production in cryptococcal meningitis
}

\section{Produção de álcool na meningite criptococócica}

Viroj Wiwanitkit

Dear editors,

Cryptococcosis is an important fungal infection affecting mainly the central nervous system (CNS). Cryptococcus neoformans is a main cause of fungal cryptococcal meningoencephalitis in immunocompromised patients, especially for cases with human immunodeficiency virus (HIV) infection $^{1}$. Mortality from HIV-associated cryptococcal meningitis remains high, owing to the inadequacy of current antifungal drugs and the complication of raised intracranial pressure ${ }^{2}$. A capsulated yeast form organism in the cerebrospinal fluid (CSF) is the general major appearance for diagnostic of the CNS cryptococcosis. Since yeast is an important composition in fermentation reaction, which leads to alcoholic production $^{3}$, a similar situation might be and can be seen in cryptococcosis. In this article, the author proposed a new hypothesis for an additional mechanism that lead to the alteration of brain function in cryptococcal meningitis, due to the effect of internal alcohol production. Theoretically, fermentation by yeast can occur at room temperature in case that there is a mixture of water, glucose ${ }^{3}$ as well as yeast, and alcohol is an important result from the fermentation.

Based on the fact that Cryptococcus spp. is a yeast-form organism, fermentation and production of alcohol can be expected. This phenomenon might be extrapolated to be another process leading to neurological deterioration. It can further imply that internal alcohol production might be an important factor leading to the alteration of brain function in cryptococcal meningitis. Indeed, cryptococcosis is an infection with chronic process before full blown of symptoms. Raised intracranial pressure might be an explanation for the alteration of consciousness in the overt phase, but it might not be a single answer for the long period of chronic deterioration of the brain function. Indeed, the amount internal alcoholic production found in this study was very low, and chronic exposure can cause problem. In chronic exposure to alcohol at a very low-level, reduced ribosomal binding to stable mRNA in brain was documented in the literature ${ }^{4}$.

\section{References}

1. Lin X, Heitman J. The biology of the Cryptococcus neoformans species complex. Ann Rev Microbiol 2006;60:69-105.

2. Bicanic T, Harrison TS. Cryptococcal meningitis. Br Med Bull 2005;72:99-118
3.

Johnston M. Feasting, fasting and fermenting. Glucose sensing in yeast and other cells. Trends Genet 1999;15:29-33.

4. Tewari S, Sweeney FM, Fleming EW. Ethanol-induced changes in properties of rat brian ribosomes. Neurochem Res 1980;5:1025-1035. 